\title{
Stress e burnout em internos de medicina geral e familiar da zona Norte de Portugal: estudo transversal
}

Paula Mendes, ${ }^{1}$ Vítor Portela Cardoso, ${ }^{2}$ John Yaphe $^{3}$

\section{RESUMO}

Objetivos: Determinar a prevalência de burnout em internos de medicina geral e familiar da zona Norte de Portugal e testar possíveis associações com fatores sociodemográficos, profissionais e stress.

Tipo de estudo: Observacional, transversal, com componente analítica.

Local: Zona Norte de Portugal.

População: Médicos internos de medicina geral e familiar.

Métodos: Amostra de conveniência constituída pelos internos presentes nas reuniões das direções de internato entre 3 de novembro a 11 de dezembro de 2009. Aplicámos o Questionário de Stress nos Profissionais de Saúde, o inventário de burnout de Maslach e colhemos dados sociodemográficos e profissionais. Calculámos a prevalência de burnout e testámos associações entre o stress laboral e o burnout.

Resultados: Estudámos uma amostra de 210 internos (68\% da população), dos quais $72 \%$ eram mulheres. A taxa de resposta foi de $92,5 \%$. A maioria dos internos referiu stress moderado $(n=127,60,5 \%)$, sobretudo nas ações de formação e em lidar com os pacientes. A prevalência de burnout variou entre 8,6\% (IC95\% 4,8-12,4\%) e 34,3\% (IC95\% 27,9-40,7\%), consoante a definição utilizada. Observámos que 29,8\% (IC95\% 23,6-36,0\%) dos internos apresentavam exaustão emocional (EE) elevada, 17,7\% (IC95\% 12,5-22,9\%) despersonalização (DP) elevada e 43,4\% (IC95\% 36,7-50,1\%) baixa realização pessoal (RP). A DP foi significativamente maior nos homens $(p<0,001)$ e naqueles que não tinham escolhido a medicina geral e familiar como primeira opção $(p<0,001)$. O nível de stress associou-se significativamente com as três dimensões do burnout $(\mathrm{EE} p<0,001, \mathrm{DP} p<0,001$ e RP $p=0,02)$. Além disso, os seis domínios de stress associaram-se com a EE $(p<0,01)$, cinco com a DP $(p<0,001)$ e o domínio carreira e remuneração com a RP $(p<0,01)$.

Conclusões: Este estudo encontrou uma prevalência estimada de burnout em internos de medicina geral e familiar similar a outros estudos. Os resultados obtidos sublinham a importância na adoção de estratégias de prevenção neste grupo vulnerável.

Palavras-chave: Burnout; Stress psicológico; Medicina geral e familiar; Internato.

\section{INTRODUÇÃO}

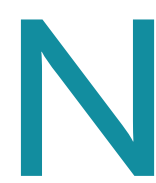

os últimos anos têm sido realizados diversos estudos a nível mundial sobre stress ocupacional e burnout em médicos internos de medicina geral e familiar (MGF), mas pouco se sabe sobre este fenómeno em Portugal. ${ }^{1-2}$

A síndroma de burnout, descrita pela primeira vez na década de 70 por Freudenberg, é caracterizada por três dimensões independentes que podem aparecer associadas: a exaustão emocional (EE) elevada, caracteri- zada pela perda de capacidade e/ou de recursos emocionais para lidar com o outro; a despersonalização (DP) elevada, que diz respeito ao desenvolvimento de atitudes de indiferença e cinismo em relação aos outros; e a baixa realização profissional (RP) que representa

\footnotetext{
1. Médica Assistente de Medicina Geral e Familiar. ARS Norte

2. Médico Assistente de Medicina Geral e Familiar. UCSP Esporões, ACES Cávado I - Braga, ARS Norte

3. Professor Associado. Instituto de Ciências da Vida e Saúde (ICVS), Escola de Ciências da Saúde, Universidade do Minho e ICVS / 3B Laboratório Associado.
} 
um estado de insatisfação do qual resultam sentimentos de incompetência e baixa auto-estima..$^{3-4} \mathrm{O}$ burnout é a consequência a uma resposta ao stress laboral crónico, contudo não deve ser confundido com este. ${ }^{5} \mathrm{O}$ stress ocupacional reflete um desequilíbrio entre as exigências do trabalho e as capacidades, recursos ou necessidades do trabalhador. ${ }^{6-7}$

A Organização Mundial da Saúde (OMS) afirma que a síndroma de burnout é uma das principais doenças do Mundo Ocidental, sendo mais prevalente nas profissões que envolvem um contacto interpessoal bem como atenção intensa e prolongada a pessoas em situação de dependência, de que são exemplo os médicos, enfermeiros ou assistentes sociais. ${ }^{3,5,8}$ Apesar da dificuldade em estimar a magnitude deste fenómeno, a OMS admite que pode afetar até $40 \%$ dos médicos a um nível suficiente para comprometer o bem-estar pessoal ou o desempenho profissional, sendo que a prevalência tem vindo a aumentar para níveis preocupantes. ${ }^{9-10}$

Não é unânime qual a especialidade médica mais afetada pelo burnout; ;11-13 $^{11}$ no entanto, a prevalência em médicos de MGF parece ser elevada devido a características específicas, como o elevado número de consultas por semana, o trabalho individual, os reduzidos tempos de consulta, a relação médico-doente mais próxima, as exigências dos doentes e o trabalho burocrático. ${ }^{14-17}$

A perceção do stress é subjetiva e tem um impacto diferente de indivíduo para indivíduo. Os fatores etiológicos do burnout dividem-se em características individuais, como o tipo de personalidade e avaliação cognitiva, autoestima, perfecionismo e uso de estratégias de coping; em características do trabalho, características organizacionais e características familiares/sociais. ${ }^{6,18-22}$ Apesar de não estar esclarecido o impacto de cada um destes fatores na síndroma de burnout, está demonstrada uma maior associação com os fatores profissionais do que com os sociodemográfi$\cos ^{10,23-24}$

Existe uma preocupação crescente nesta área devido ao seu impacto não só a nível individual, como também profissional e organizacional. ${ }^{7}$ Alguns estudos demonstraram inclusive uma associação entre profissionais sob stress e a sua performance ou até erros médicos percecionados quer pelos médicos quer pelos doentes. $^{25-28}$
Os internos de MGF foram o alvo deste estudo, uma vez que alguns estudos obtiveram prevalências de burnout elevadas nesta categoria profissional. Neste período ocorre uma transição das expectativas idealistas desenvolvidas durante a formação para o mundo real da prática quotidiana, começando o jovem profissional a tomar consciência de que as recompensas pessoais, profissionais e económicas que recebe podem não corresponder às esperadas. ${ }^{28-29} \mathrm{Além}$ disso, é caracterizado por aumento da responsabilidade profissional, sobrecarga de trabalho, competição interpares associado a isolamento social, fadiga, privação do sono e receio de cometer erros. ${ }^{13,28,30}$ Assim, os objetivos deste estudo foram determinar a prevalência de burnout em internos de MGF da zona Norte de Portugal (IMGFZN) e testar possíveis associações deste com variáveis sociodemográficas e profissionais. Ao identificar populações vulneráveis poderá ser possível desenhar estratégias dirigidas e apropriadas de prevenção e intervenção.

\section{MÉTODOS}

Realizámos um estudo observacional, transversal, com componente analítica, cuja população alvo foram os IMGFZN que se encontravam distribuídos pelos distritos de Braga, Bragança, Porto, Viana do Castelo, Vila Real e Aveiro. Segundo os dados da Administração Regional de Saúde do Norte (ARSN), em setembro de 2009, essa população correspondia a 311 internos. Realizámos um estudo piloto a cinco internos a fim de aferir a aplicabilidade e exequibilidade do questionário e identificar obstáculos não previstos no protocolo de investigação. Posteriormente utilizámos uma amostra de conveniência constituída pelos internos presentes nas 11 reuniões das direções de internato da zona Norte e que aceitaram participar voluntariamente no estudo, no período compreendido entre 3 de novembro e 11 de dezembro de 2009. A recolha dos dados foi realizada através de um questionário de autopreenchimento, confidencial e anónimo.

Este estudo de investigação foi autorizado pela Coordenação de Internato de MGF da zona Norte e aprovado pela comissão de ética da ARSN. Todos os participantes assinaram o respetivo termo de consentimento informado.

O questionário encontrava-se dividido em três secções. A secção A era constituída pelo Questionário de 
Stress nos Profissionais de Saúde (QSPS). ${ }^{31-32}$ Este instrumento possui 22 itens relativos a potenciais fontes de stress associadas à profissão, respondidos numa escala tipo Likert de 5 pontos (0 - Nenhum stress a 4 - Elevado stress). Esses itens distribuíam-se em seis domínios: lidar com os doentes, relações profissionais, excesso de trabalho, carreira e remuneração, ações de formação e problemas familiares.

A pontuação de cada domínio foi obtida através da média de todos os itens de cada domínio.

Para avaliação do burnout aplicámos o Inventário de Burnout de Maslach (MBI-HSS) - secção B - desenvolvida por Christina Maslach. ${ }^{33}$ Utilizámos uma versão traduzida para português que incluía 22 itens avaliados numa escala tipo Likert de 7 pontos $(0$ - Nunca a 6 - Todos os dias), sendo distribuídos pelas três dimensões do burnout (EE, DP e RP). ${ }^{34-35}$ À semelhança de outros estudos anteriores e pelo facto de não existirem dados aferidos para a população portuguesa para classificar os participantes como tendo níveis elevados, médios ou baixos em cada dimensão do burnoutadotámos os seguintes pontos de corte: score EE: baixa $\leq 13$, média 14-26 e elevada $\geq 27$; score DP: baixa $\leq 5$, média 6-9 e elevada $\geq 10$; score RP (pontua de modo inverso): baixa $\leq 33$, média 32-39 e elevada $\geq 40 .{ }^{10,36}$ Para a definição da síndroma de burnout adotámos as seguintes definições: (1) elevados níveis de EE e DP combinada com RP baixa; ${ }^{37}$ e (2) EE e/ou DP elevada. ${ }^{38-39}$

Por último, a secção $C$ visava obter dados demográficos (idade, sexo, estado civil, número de filhos, se o(a) companheiro(a) ou marido/esposa se encontra na mesma carreira profissional) e variáveis profissionais (ano de especialidade, instituição da graduação, local de trabalho, número de horas de trabalho semanais, satisfação profissional, motivação inicial e atual para a especialidade, satisfação com o programa de internato, satisfação com o local de formação, se MGF foi a primeira opção como especialidade, se voltariam a escolher MGF e local de formação).

Para a análise dos resultados recorremos ao programa Statistical Package for the Social Sciences ${ }^{\circledR}$ (SPSS, versão 17.0 for Windows ${ }^{\oplus}$ SPSS Inc., 1989-2008). Realizámos uma análise descritiva dos dados, bem como analítica. Na análise bivariável utilizámos testes não paramétricos (qui-quadrado de Pearson, Mann-Whitney, Kruskall-Wallis e correlação de Spearman), uma vez que nenhuma variável quantitativa apresentou uma distribuição normal, segundo o coeficiente de Kolmogorov-Smirnov. Considerámos existir significância estatística para valores de $p \leq 0,05$ com um intervalo de confiança (IC) de 95\%.

Excluímos da amostra os participantes do estudo piloto, todos os que se recusaram a participar e aqueles que não responderam a $\geq 20 \%$ de perguntas do questionário total. ${ }^{40}$ No caso das escalas QSPS e MBI-HSS excluímos apenas para análise das respetivas escalas os questionários em que uma ou mais pergunta(s) não fosse(m) respondida(s), a fim de não interferir na pontuação total de cada domínio.

Assumindo uma prevalência esperada de burnout de 30\%, uma precisão de 0,05 e um IC de 95\%, a dimensão amostral necessária para uma amostra aleatória da população seria de 159, segundo o programa Epi Info $^{\mathrm{TM}} 7$. Os autores adotaram as normas STROBE para a redação do presente estudo. ${ }^{41}$

\section{RESULTADOS}

Da amostra de 227 médicos internos foram incluídos 210, o correspondente a uma taxa de resposta de $92,5 \%$ (Figura 1).

\section{Caracterização sociodemográfica da amostra}

Na amostra estudada, 71,9\% ( $n=151)$ (IC95\% 65,8$78,0 \%$ ) dos internos era do sexo feminino, com uma idade mediana de 28 anos, compreendidas entre 25 e 54 anos. A distribuição por sexo na amostra era semelhante à da população total, onde a proporção de indivíduos do sexo feminino era de $67,8 \%$. A proporção de internos do primeiro ano na nossa amostra $(48,7 \%$, IC95\% 41,9-55,5\%) era ligeiramente superior à verificada na população $(41,4 \%)$ e a proporção de internos do terceiro ano na nossa amostra (20,1\%, IC95\% 14,7$25,5 \%)$ era ligeiramente inferior à da população em estudo (29\%).

A caracterização sociodemográfica encontra-se descrita no Quadro I.

\section{Análise psicométrica da escala MBI-HSS}

Na análise da consistência interna, o valor do Alfa de Cronbach para a EE e RP foram elevados, respetivamente 0,884 e 0,821 e o da DP obteve um coeficiente considerado médio (0,691). Eliminando o pior item, os 
valores do Alfa de Cronbach corrigidos não sofriam alterações relevantes.

\section{Satisfação profissional}

A maioria dos internos classificou como boa ou muito boa a sua satisfação profissional ( $n=156,75 \%)$ e a sua satisfação com o local de formação $(n=150$, $72,1 \%)$. No entanto, os internos parecem insatisfeitos quanto ao programa de formação, sendo classificada como "muito baixa, baixa ou suficiente" em $54,7 \%(n=114)$ dos questionados (Figura 2).

A MGF foi referida como a primeira opção na escolha da especialidade em $70,8 \%$ ( $n=148)$ dos questionários. Estes internos apresentaram significativamente melhor satisfação profissional $(78,8 \%)$ do que os que não escolheram MGF como primeira opção $(64,6 \%)(p=0,046)$. Apesar disso, $78,1 \%$ dos internos $(n=164)$ voltariam a escolher MGF como especialidade e um pouco menos ( $n=145$, $70 \%$ ) voltaria a escolher o mesmo local de formação.

\section{Fontes de stress - Escala QSPS}

A maioria dos internos referiu stress moderado ( $n=127,60,5 \%)$. Avaliando os seis domínios da escala
População em estudo $n=311$

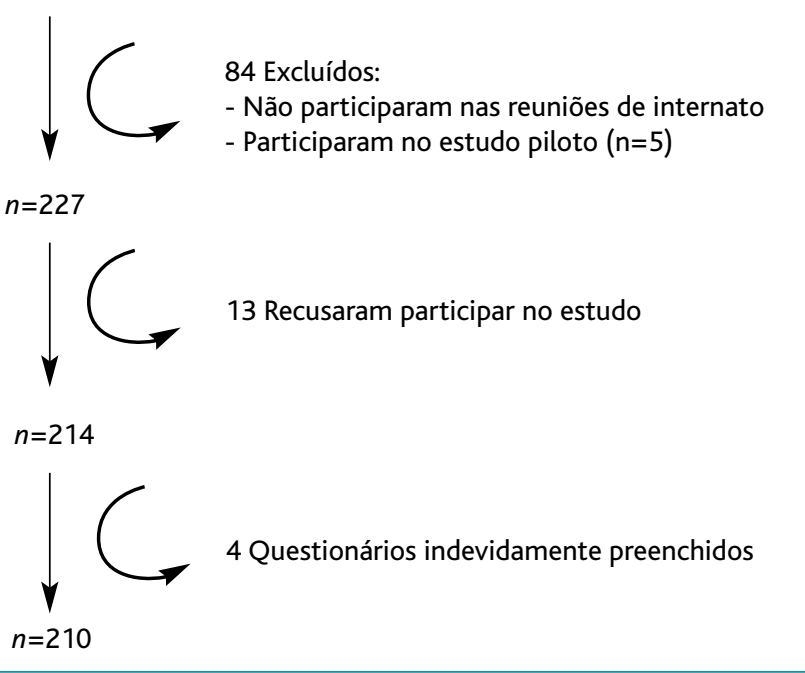

Figura 1. Fluxograma da amostragem.

QSPS, os internos referiram sentir maior stress nas ações de formação e em lidar com os pacientes, especialmente as participantes do sexo feminino $(p<0,001 \mathrm{e}$ $p<0,004$, respetivamente). No Quadro II encontram-se descritos os resultados nos seis domínios da escala QSPS.

Analisando cada uma das 22 fontes de stress da escala, as três percecionadas como causadoras de maior

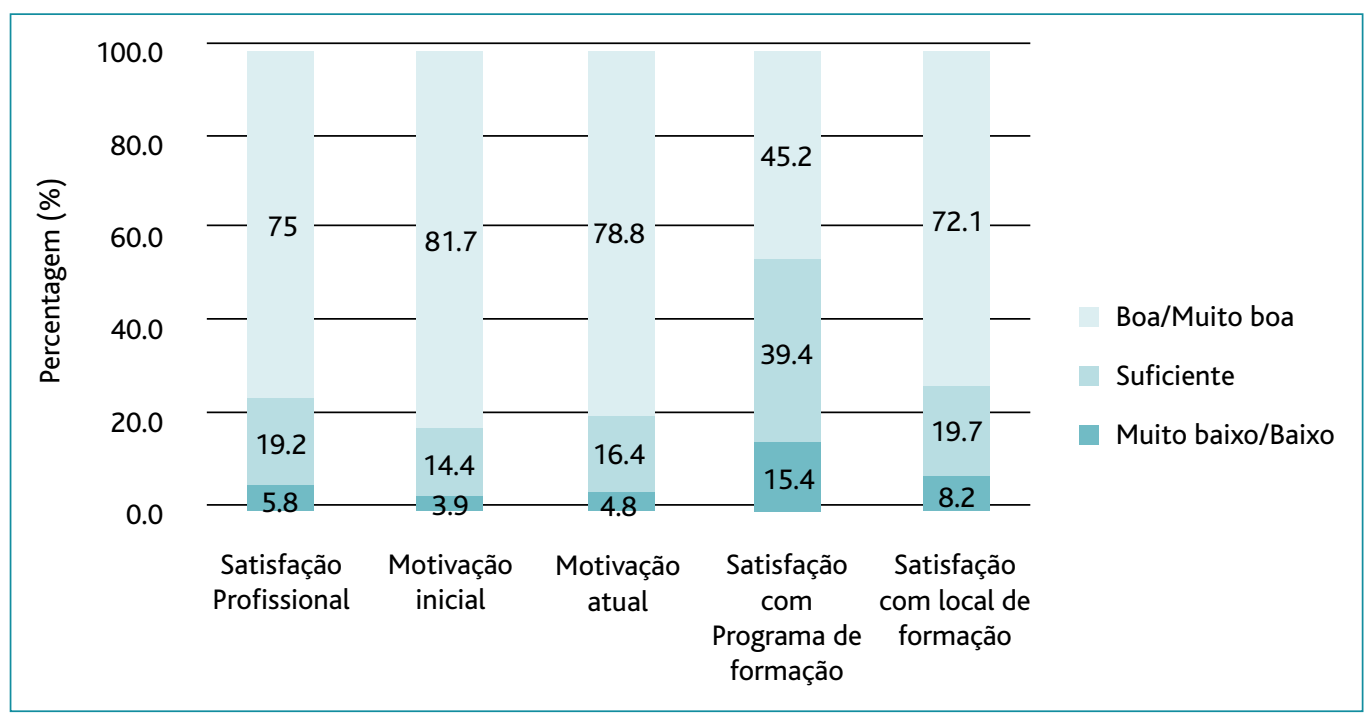

Figura 2. Caracterização do grau de satisfação profissional, motivação e satisfação com o internato de MGF. 


\begin{tabular}{|c|c|c|c|c|}
\hline & \multicolumn{2}{|c|}{$n$} & $\%$ & $\begin{array}{c}\text { Amostra } \\
\text { válida/em } \\
\text { falta }\end{array}$ \\
\hline \multicolumn{5}{|l|}{ Sexo } \\
\hline Feminino & \multicolumn{2}{|c|}{151} & 71,9 & $210 / 0$ \\
\hline Masculino & \multicolumn{2}{|c|}{59} & 28,1 & \\
\hline \multicolumn{5}{|l|}{ Estado civil } \\
\hline Solteiro & \multicolumn{2}{|c|}{133} & 63,3 & $210 / 0$ \\
\hline Casado & \multicolumn{2}{|c|}{62} & 29,7 & \\
\hline União facto & \multicolumn{2}{|c|}{14} & 6,7 & \\
\hline Divorciado & \multicolumn{2}{|c|}{1} & 0,5 & \\
\hline \multicolumn{5}{|l|}{$\begin{array}{l}\text { Companheiro/cônjuge na } \\
\text { mesma carreira profissional }\end{array}$} \\
\hline Sim & \multicolumn{2}{|c|}{61} & 32,4 & $188 / 22$ \\
\hline Não & \multicolumn{2}{|c|}{111} & 59 & \\
\hline Não aplicável & \multicolumn{2}{|c|}{16} & 8,5 & \\
\hline \multicolumn{5}{|l|}{ N. ${ }^{\circ}$ filhos } \\
\hline 0 & \multicolumn{2}{|c|}{169} & 84,5 & $200 / 10$ \\
\hline$\geq 1$ & \multicolumn{2}{|c|}{31} & 15,5 & \\
\hline \multicolumn{5}{|l|}{ Ano de especialidade } \\
\hline $1 .^{\circ}$ & \multicolumn{2}{|c|}{97} & 48,7 & 199/11 \\
\hline $2 .^{\circ}$ & \multicolumn{2}{|c|}{62} & 31,2 & \\
\hline 3. & \multicolumn{2}{|c|}{40} & 20,1 & \\
\hline \multicolumn{5}{|l|}{ Instituição onde se graduou } \\
\hline FMUP & \multicolumn{2}{|c|}{84} & 40,4 & $208 / 2$ \\
\hline ICBAS & \multicolumn{2}{|c|}{59} & 28,4 & \\
\hline FCM-UNL & \multicolumn{2}{|c|}{15} & 7,2 & \\
\hline FMUC & \multicolumn{2}{|c|}{11} & 5,3 & \\
\hline ECS-UM & \multicolumn{2}{|c|}{9} & 4,3 & \\
\hline FMUL & \multicolumn{2}{|c|}{9} & 4,3 & \\
\hline FCS-UBI & \multicolumn{2}{|c|}{5} & 2,4 & \\
\hline Outra & \multicolumn{2}{|c|}{16} & 7,7 & \\
\hline Local de trabalho & & & & \\
\hline Predominante urbano & 9 & & 46,9 & $207 / 3$ \\
\hline Moderadamente urbano & 8 & & 39,1 & \\
\hline Predominantemente rural & 2 & & 14 & \\
\hline & Mediana & Média & Desvio-padrão & \\
\hline Idade (anos) & 28 & 29,04 & 4,9 & $205 / 5$ \\
\hline N. ${ }^{\circ}$ horas de trabalho semanais (h) & 60 & 61,09 & 14 & $204 / 6$ \\
\hline
\end{tabular}

Legenda: ECS-UM - Escola de Ciências da Saúde da Universidade do Minho; FCM-UNL - Faculdade de Ciências Médicas da Universidade Nova de Lisboa; FCS-UBI - Faculdade de Ciências da Saúde da Universidade da Beira Interior; FMUC - Faculdade de Medicina da Universidade de Coimbra; FMUL - Faculdade de Medicina da Universidade de Lisboa; FMUP - Faculdade de Medicina da Universidade do Porto; ICBAS - Instituto de Ciências Biomédicas Abel Salazar. stress foram: "tomar decisões onde os erros possam ter consequências graves para os doentes" ( $n=126,60 \%)$, seguido de "excesso de trabalho e/ou tarefas de carácter burocrático" ( $n=124,59,1 \%)$ e "falar/fazer apresentações em público" ( $n=116,55,2 \%)$.

\section{Prevalência de burnout}

Observámos que 29,8\% (IC95\% 23,6-36,0\%) dos internos apresentava uma EE elevada, 17,7\% (IC95\% 12,5$22,9 \%)$ uma DP elevada e $43,4 \%$ uma RP baixa (IC95\% 36,7-50,1\%). Assim, a RP foi a dimensão da síndroma de burnout mais afetada das três (Quadro III).

Calculando a mediana de cada uma das três dimensões do burnout e comparando com os valores de referência, obtivemos que a EE encontrava-se no nível médio, a DP no nível baixo e a RP no nível médio (Quadro IV).

Quanto ao número de dimensões do burnout afetadas, 58,6\% ( $n=116)$ tinha pelo menos uma dimensão afetada e 8,6\% ( $n=17)$ revelaram todas as dimensões afetadas (Figura 3).

Segundo os critérios definidos, obtivemos uma prevalência de burnout nos internos de MGF de 8,6\% $(n=17)($ IC95\% 4,8-12,4\%) para a definição 1 (EE e DP elevada + RP baixa) e 34,3\% ( $n=68)$ (IC95\% 27,9-40,7\%) para a definição 2 (EE e/ou DP elevada). 
Burnout e variáveis sociodemográficase profissionais

Os resultados da análise inferencial entre as características sociodemográficas e profissionais com EE elevado, DP elevado e RP baixo encontram-se no Quadro V. Os homens estavam mais despersonalizados do que as mulheres $(p=0,001)$. A elevada EE foi significativamente maior nos internos com menor satisfação profissional $(p<0,001)$, menor motivação atual ( $p<0,001)$, menor satisfação com o programa de formação ( $p=0,003)$ ou com o local de formação $(p<0,001)$. A DP revelou-se significativamente elevada em internos com menor satisfação profissional $(p=0,05)$, menor motivação atual $(p<0,001)$, menor satisfação com local de formação $(p=0,016)$ e com o facto de não ter escolhido MGF como primeira opção ( $p=0,019$ ). Por último, a RP foi significativamente menor nos internos com menor motivação inicial ( $p=0,015)$ ou atual $(p=0,011)$.

No Quadro VI representa-se a análise bivariável entre as três dimensões do burnout e a escala QSPS. Observou se que a pontuação obtida na EE e DP aumentava progressivamente com o aumento do nível de stress $(p<0,001 \mathrm{e}$ $p<0,001)$ bem como com o aumento da sua frequência ( $p<0,001$ e 0,001). Adicionalmente, quanto maior o nível de stress percecionado, menor era a $\operatorname{RP}(p=0,02)$.

O coeficiente de correlação de Spearman demonstrou uma associação positiva e estatisticamente significativa da pontuação obtida na EE com todos os domínios da QSPS ( $p<0,001)$. De igual modo, níveis mais elevados de DP associaram-se a maiores níveis de stress

\begin{tabular}{|c|c|c|c|c|}
\hline Burnout & $\mathrm{EE}(n / \%)$ & $\mathrm{DP}(n / \%)$ & $\operatorname{RP}(n / \%) *$ & $\begin{array}{c}\text { Amostra válida/ } \\
\text { em falta }\end{array}$ \\
\hline Nível baixo & $40(20,2 \%)$ & $112(56,6 \%)$ & $86(43,4 \%)$ & $198 / 12$ \\
\hline Nível médio & 99 (50\%) & $51(25,8 \%)$ & $60(30,3 \%)$ & \\
\hline Nível elevado & $59(29,8 \%)$ & 35 (17,7\%) & $52(26,3 \%)$ & \\
\hline
\end{tabular}

*Score baixo ( $\leq 33$ ) na RP significa Nível de burnout elevado - porque esta dimensão pontua de modo inverso.

Legenda: EE - Exaustão Emocional; DP - Despersonalização; RP - Realização Profissional.

\begin{tabular}{|c|c|c|c|c|}
\hline & $\begin{array}{c}\text { Total - mediana } \\
\text { (mínimo - máximo) }\end{array}$ & $\begin{array}{l}\text { Homens } \\
\text { (mediana) }\end{array}$ & $\begin{array}{l}\text { Mulheres } \\
\text { (mediana) }\end{array}$ & $\begin{array}{c}\text { Amostra válida/ } \\
\text { em falta }\end{array}$ \\
\hline Score EE & $21(3-42)$ & 19 & 21 & $198 / 12$ \\
\hline Score DP & $5(0-18)$ & 6 & 5 & \\
\hline Score RP & $35(0-48)$ & 35 & 35 & \\
\hline
\end{tabular}

Legenda: EE - Exaustão Emocional; DP - Despersonalização; RP - Realização Profissional.

nos domínios: lidar com pacientes ( $p=0,01)$, excesso de trabalho $(p<0,001)$, problemas familiares $(p<0,001)$, carreira e remuneração $(p<0,001)$ e relações profissionais $(p<0,001)$. A pontuação da RP variou inversamente com a “carreira e remuneração" ( $p=0,002)$. 


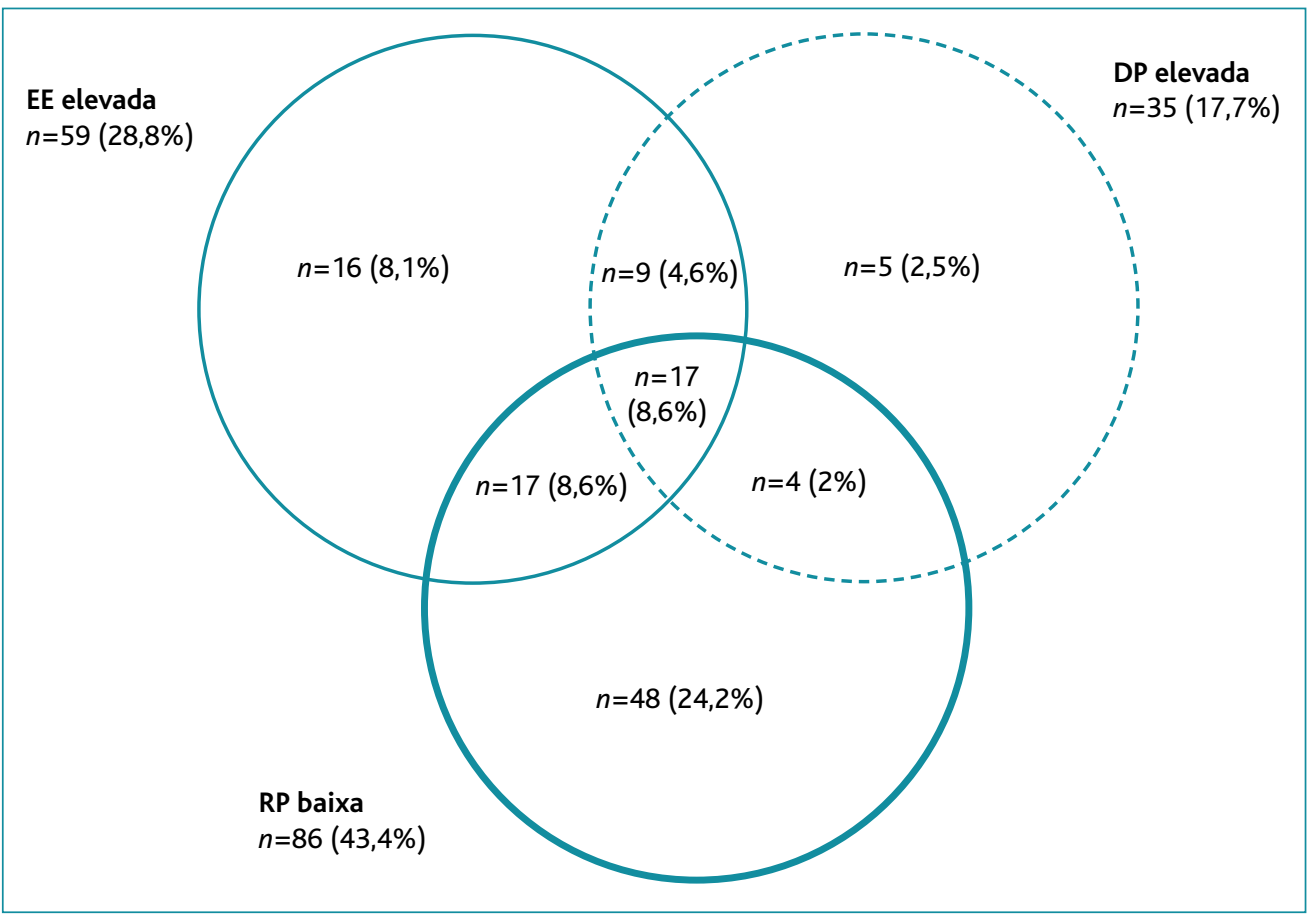

Figura 3. Diagrama de Venn da distribuição dos participantes pelas dimensões de burnout (EE, DP, RP). Legenda: EE - Exaustão Emocional; DP - Despersonalização; RP - Realização Profissional.
Os valores da prevalência de burnout são variáveis entre as investigações realizadas, o que poderá ser o reflexo de vários fatores. Por um lado, não existe uma forma consensual de medir o burnout. Alguns estudos afirmam que basta ter uma das dimensões afetadas para definir a síndroma, ${ }^{42-43}$ enquanto outros exigem um trio constituído por EE e DP elevadas e RP baixa. ${ }^{44-45}$ Por outro lado, a ausência de valores normativos para a população portuguesa pode subestimar a prevalência de
I-HSS ter sido já alvo de burnout. Apesar de a escala MBI-HSS ter sido já alvo de
trabalhos de tradução e validação para Portugal, ${ }^{34-35}$ seria importante usar valores de referência adaptados à cultura do país e às diferentes populações estudadas (médicos vs médicos internos). No processo de verificação das propriedades psicométricas, a MBI-HSS revelou ter uma boa consistência interna na generalidade das dimensões que pretendeu medir, revelando ser um instrumento fiável, sensível e válido para medir o burnout na população estudada.

As mulheres apresentaram valores médios mais elevados de EE e níveis mais baixos de RP do que os homens, porém, sem uma diferença estatisticamente significativa. Adicionalmente, os internos homens eram os que estavam mais despersonalizados, como já verificado noutros estudos. ${ }^{10,46-47}$ Todavia, no estudo de Gisela Marcelino foram os homens que apresentaram maiores níveis de EE e DP associados a menor RP, realçando o envolvimento da mulher na família e o papel dos filhos como fator protetor. ${ }^{36}$ Os dados relativos às diferenças entre sexo são importantes pelo facto de se verificar um aumento progressivo do sexo feminino na população médica. ${ }^{48}$ 


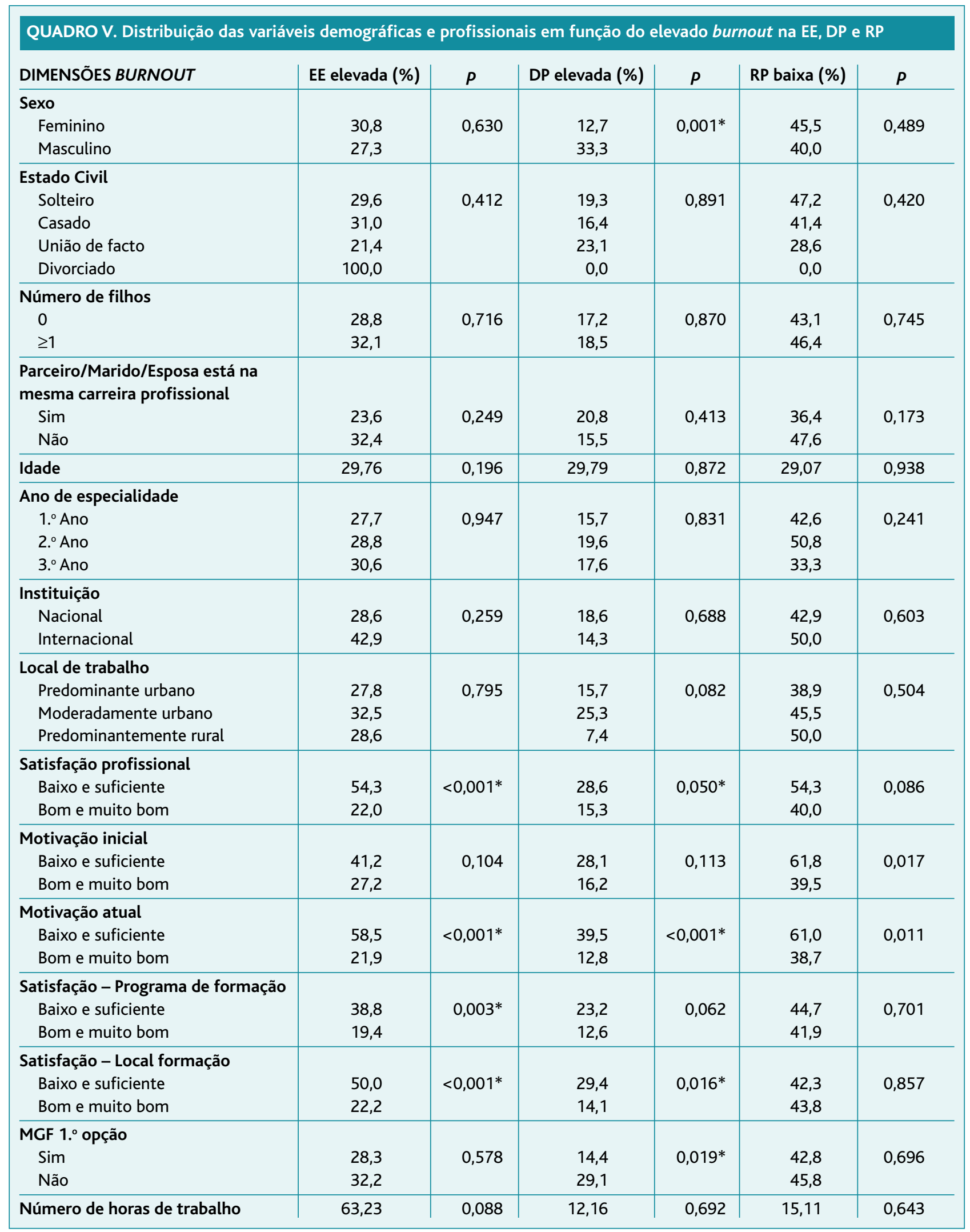

* $\mathrm{p} \leq 0,05$. Teste Qui-quadrado de Pearson para as variáveis nominais e o teste de Mann-Whitney para as variáveis quantitativas (idade e número de horas de trabalho).

Legenda: EE - Exaustão Emocional; DP - Despersonalização; RP - Realização Profissional. 


\begin{tabular}{|c|c|c|c|c|c|c|c|}
\hline & \multirow[b]{2}{*}{$n$} & \multicolumn{2}{|c|}{$\mathrm{EE}$} & \multicolumn{2}{|c|}{ DP } & \multicolumn{2}{|l|}{ RP } \\
\hline & & Mediana & $p$ & Mediana & $p$ & Mediana & $p$ \\
\hline \multicolumn{8}{|l|}{ Nível de stress } \\
\hline Nenhum & 1 & 3 & $<0,001^{*}$ & 4 & $<0,001^{*}$ & 33 & $0,02^{*}$ \\
\hline Pouco & 36 & 12,19 & & 3,33 & & 36,97 & \\
\hline Moderado & 119 & 20,81 & & 5,51 & & 37,7 & \\
\hline Bastante & 35 & 29,80 & & 7,46 & & 31,94 & \\
\hline Elevado & 7 & 33,14 & & 8,43 & & 31,86 & \\
\hline \multicolumn{8}{|l|}{ Frequência de stress } \\
\hline Nenhum & 2 & 12,5 & $<0,001^{*}$ & 7 & $0,001^{*}$ & 37 & 0,169 \\
\hline Algumas vezes no ano & 15 & 11,33 & & 3,93 & & 33,8 & \\
\hline Uma vez ao mês & 45 & 16 & & 3,78 & & 36,26 & \\
\hline Uma vez à semana & 107 & 22,7 & & 5,94 & & 32,94 & \\
\hline \multirow[t]{2}{*}{ Todos os dias } & 32 & 30,25 & & 7,09 & & 34,38 & \\
\hline & $n$ & $\begin{array}{c}\text { Coeficiente } \\
\text { de } \\
\text { correlação }\end{array}$ & $P$ & $\begin{array}{c}\text { Coeficiente } \\
\text { de } \\
\text { correlação }\end{array}$ & $P$ & $\begin{array}{c}\text { Coeficiente } \\
\text { de } \\
\text { correlação }\end{array}$ & $P$ \\
\hline Ações de formação & 191 & 0,314 & $<0,001^{*}$ & 0,091 & 0,209 & $-0,023$ & 0,748 \\
\hline Lidar com pacientes & & 0,334 & $<0,001^{*}$ & 0,185 & $0,01^{*}$ & $-0,034$ & 0,638 \\
\hline Excesso de trabalho & & 0,483 & $<0,001^{*}$ & 0,360 & $<0,001^{*}$ & $-0,044$ & 0,544 \\
\hline Problemas familiares & & 0,412 & $<0,001^{*}$ & 0,279 & $<0,001^{*}$ & $-0,029$ & 0,688 \\
\hline Carreira e remuneração & & 0,341 & $<0,001^{*}$ & 0,274 & $<0,001^{*}$ & $-0,225$ & $0,002 *$ \\
\hline Relações profissionais & & 0,432 & $<0,001^{*}$ & 0,280 & $<0,001^{*}$ & $-0,124$ & 0,088 \\
\hline
\end{tabular}

${ }^{*} p \leq 0,05$. Teste Kruskall-Wallis

Legenda: EE - Exaustão Emocional; DP - Despersonalização; DP - Desvio-padrão; QSPS - Questionário de Stress nos Profissionais de Saúde; RP - Realização Profissional.

Quanto à satisfação profissional, 75\% dos internos classificou-a como boa ou muito boa e apenas 5,8\% como baixa ou muito baixa. Um estudo mais recente, realizado também em IMGZN, obteve dados semelhantes com apenas $5 \%$ dos internos a mostrarem-se insatisfeitos ou muito insatisfeitos. ${ }^{49}$ Por outro lado, a menor satisfação profissional, menor motivação, menor satisfação com o programa de formação e com o local de formação associaram-se a algumas dimensões do burnout. Em 2005, uma meta-análise concluiu que indivíduos com níveis de satisfação laboral mais baixos apresentavam níveis de burnout mais elevados, menor autoestima e mais problemas psicológicos, como depressão e ansiedade..$^{50}$
Cerca de um terço dos internos referem que o seu/sua companheiro(a) ou marido/esposa está na mesma carreira profissional. Estudos anteriores demonstraram que estar casado com outro médico tinha impacto em termos familiares e profissionais, uma vez que eram mais felizes, divorciavam-se menos, mas estariam sob maior stress e mais sujeitos a burnout. . $^{17,51}$ Embora sem diferenças significativas, no nosso estudo os internos com parceiro(a) na mesma carreira estavam menos exaustos e mais realizados pessoalmente, apesar de mais despersonalizados.

Tem sido questionado se a escolha pela especialidade médica de MGF não será influenciada pela dificuldade de acesso a especialidades hospitalares. É prová- 
vel que até certo ponto isto se verifique, uma vez que o maior volume de escolhas de MGF centra-se nas classificações mais baixas, ${ }^{52}$ podendo a escolha pela MGF ser uma segunda opção para alguns. ${ }^{50,53}$ No nosso estudo verificou-se que os cerca de $30 \%$ dos internos que não escolheram MGF como primeira opção tinham significativamente uma menor satisfação profissional e uma maior despersonalização. No entanto, apenas $8,6 \%$ admitiram não voltar a escolher MGF. Um estudo posterior, realizado entre 2011 e 2013, revelou que $76 \%$ dos IMGFZN escolheram MGF como primeira opção, estando estes mais satisfeitos com o internato do que os que não viram esta especialidade como preferencial $(p=0,001) .{ }^{49}$

Os internos que referiram percecionar elevados níveis de stress estavam sob elevada exaustão emocional e encontravam-se menos realizados pessoalmente, segundo os valores de referência nas dimensões EE $(\geq 27)$ e RP ( $\leq 33)$. As fontes de stress da escala QSPS estiveram particularmente associadas às dimensões de burnout EE e DP, sendo que apenas o domínio "carreira e remuneração" se associou simultaneamente com as três dimensões afetadas. Sendo o burnoutuma consequência ao stress laboral crónico, a associação entre estes dois problemas não foi surpreendente. ${ }^{5}$

O nosso estudo apresenta algumas limitações que não podem ser negligenciadas. A amostra utilizada foi de conveniência, podendo introduzir um viés de seleção. Apesar disto, realçamos o facto de que a amostra estudada foi superior à amostra necessária para ser representativa da população. É possível, porém, que os internos que não estiveram presentes nas reuniões estejam sob maior stress e com mais burnout. A altura da recolha dos dados (novembro e dezembro de 2009) pode ter ainda sobrestimado a prevalência de burnout, uma vez que é uma fase do internato mais exigente com a proximidade das avaliações anuais. Por outro lado, a utilização de um questionário de autopreenchimento está sujeita a erros de interpretação e poderá ter havi- do tendência para os internos responderem o que é socialmente correto (desejabilidade social). Adicionalmente, os autores consideram que o questionário é relativamente extenso. Outras limitações poderão estar relacionadas com os quatro questionários excluídos da análise por preenchimento indevido e em que, apesar de identificados os dados omissos, não foi realizada a sua análise estatística.

Apesar das limitações referidas, a que se adiciona o atraso na publicação dos resultados, pretendemos realçar um problema que consideramos ser atual. Desde a colheita dos nossos dados até à publicação deste artigo ocorreram importantes alterações no internato de MGF em Portugal, como a duração estendida para quatro anos e a recente alteração da avaliação final do internato médico. ${ }^{54} \mathrm{~A}$ crise financeira também trouxe grandes implicações a nível socioeconómico. Por estes motivos, consideramos ser fundamental estudar esta temática à luz do panorama atual, identificando outras possíveis fontes de burnout ou mesmo diferenças territoriais no país, de acordo com os diferentes programas de internato. No futuro, poderá ainda ser alvo de análise a relação de fatores individuais (como a personalidade) com esta síndroma.

Gostaríamos ainda de salientar o facto de termos conseguido, num curto período de tempo, uma amostra representativa quanto ao sexo, constituída por internos de todas as direções de internato do Norte de 
Portugal e que representou $68 \%$ da população total, com uma taxa de adesão ao estudo de $92,5 \%$. Por outro lado, além do burnout, identificámos fontes de stresslaboral que lhe estavam associadas, o que poderá delinear as áreas prioritárias de prevenção e intervenção.

Os resultados que obtivemos são fontes importantes de reflexão. Parece-nos importante consciencializar os internos dessa realidade, pois é frequente os médicos adotarem uma postura de negação (estilo de coping ineficaz) relativamente a estes problemas relacionados com o burnout. ${ }^{55-56}$ Foi proposto um modelo de intervenção integrada do burnout, o qual completa diferentes focos e níveis de ação em vários domínios. ${ }^{57} \mathrm{~A}$ formação pré-graduada parece ser uma fase particularmente importante na prevenção desta síndroma, dado que é nesta altura que os futuros médicos aprendem as características da sua profissão e adquirem hábitos que os tornarão suscetíveis ao burnout. .9,58 $^{40}$

Em conclusão, os resultados colocaram os internos de MGF num grupo de risco, sendo necessário delinear precocemente estratégias de prevenção e intervenção.

\section{AGRADECIMENTOS}

Os autores gostariam de agradecer a colaboração da Coordenação de Internato de MGF da zona Norte, aos diretores de internato e a todos os colegas internos que colaboraram voluntariamente neste estudo, sem os quais não teria sido possível concretizá-lo.

\section{REFERÊNCIAS BIBLIOGRÁFICAS}

1. Galam E, Komly V, Le Tourneur A, Jund J. Burnout among French GPs in training: a cross-sectional study. Br J Gen Pract. 2013;63(608):e21724.

2. Dyrbye LN, West CP, Satele D, Boone S, Tan L, Sloan J, et al. Burnout among U.S. medical students, residents, and early career physicians relative to the general U.S. population. Acad Med. 2014;89(3):443-51.

3. World Health Organization. Primary prevention of mental, neurological and psychosocial disorders [Internet]. Geneva: WHO; 1998. ISBN 924154516X.Available from: http://apps.who.int/iris/bitstream/10665/ 42043/1/924154516X_eng.pdf

4. Maslach $C$, Jackson SE, Leiter MP. Maslach Burnout inventory manual. 3rd ed. Palo Alto, CA: Consulting Psychologist Press; 1996.

5. Maslach C, Schaufeli WB, Leiter MP. Job burnout. Annu Rev Psychol. 2001;52:397-422.

6. Uva AS, Leite ES. Stress relacionado com o trabalho. Saúde e Trabalho. 2007;6:25-42.

7. Milczarek M, Schneider E, Rial-González E. OSH in figures: stress at work - facts and figures [Internet]. Luxembourg: Office for Official Publications of the European Communities; 2009. ISBN 9789291912247. Avai- lable from: https://osha.europa.eu/pt/tools-and-publications/publications/reports/TE-81-08-478-EN-C_OSH_in_figures_stress_at_work

8. Shanafelt TD, Boone S, Tan L, Dyrbye LN, Sotile W, Satele D, et al. Burnout and satisfaction with work-life balance among US physicians relative to the general US population. Arch Intern Med. 2012;172(18): 1377-85.

9. Devi S. Doctors in distress. Lancet. 2011;377(9764):454-5.

10. Soler JK, Yaman H, Esteva M, Dobbs F, Asenova RS, Katic M, et al. Burnout in European family doctors: the EGPRN study. Fam Pract. 2008;25(4):245-65.

11. McCray LW, Cronholm PF, Bogner HR, Gallo JJ, Neill RA. Resident physician burnout: is there hope? Fam Med. 2008;40(9):626-32.

12. Martini S, Arfken CL, Churchill A, Balon R. Burnout comparison among residents in different medical specialties. Acad Psychiatry. 2004;28(3):240-2.

13. IsHakWW, Lederer S, Mandili C, Nikravesh R, Seligman L, Vasa M, et al. Burnout during residency training: a literature review. J Grad Med Educ. 2009; 1(2):236-42.

14. Frasquilho MA. Medicina, uma jornada de 24 horas? Stress e burnout em médicos: prevenção e tratamento [Medicine: a 24 hours journay?]. Rev Port Saúde Pública. 2005;23(2):89-98. Portuguese

15. Schattner PL, Coman GJ. The stress of metropolitan general practice. Med J Aust. 1998;169(3):133-7.

16. Royal College of General Practitioners. Stress and general practice. London: RCGP; 2005.

17. Rout $U$. Stress among general practitioners and their spouses: a qualitative study. Br J Gen Pract. 1996;46(404):157-60.

18. Souza WC, Silva AM. A influência de fatores de personalidade e de organização do trabalho no burnout em profissionais de saúde [The influence of personality and job factors on burnout among health professionals]. Estud Psicol. 2002;19(1):37-48. Portuguese

19. McManus IC, Keeling A, Paice E. Stress, burnout and doctors' attitudes to work are determined by personality and learning style: a twelve year longitudinal study of UK medical graduates. BMC Med. 2004;2:29.

20. Cohen JC, Leung Y, Fahey M, Hoyt L, Sinha R, Cailler L, et al. The happy docs study: a Canadian Association of Internes and Residents well-being survey examining resident physician health and satisfaction within and outside of residency training in Canada. BMC Res Notes. 2008;1:105.

21. Lee FJ, Stewart M, Brown JB. Stress, burnout, and strategies for reducing them: what's the situation among Canadian family physicians? Can Fam Physician. 2008;54(2):234-5.

22. Lee FJ, Brown JB, Stewart M. Exploring family physician stress: helpful strategies. Can Fam Physician. 2009;55(3):288-9.

23. Ozyurt A, Hayran O, Sur H. Predictors of burnout and job satisfaction among Turkish physicians. Q J Med. 2006;99(3):161-9.

24. Al-Dubai SA, Rampal KG. Prevalence and associated factors of burnout among doctors in Yemen. J Occup Health. 2010;52(1):58-65.

25. Dewa CS, Loong D, Bonato S, Thanh NX, Jacobs P. How does burnout affect physician productivity? A systematic literature review. BMC Health Serv Res. 2014;14:325.

26. Hill JD, Smith RJ. Monitoring stress levels in postgraduate medical training. Laryngoscope. 2009;119(1):75-8.

27. van den Hombergh P, Künzi B, Elwyn G, van Doremalen J, Akkermans $R$, Grol R, et al. High workload and job stress are associated with lower 
practice performance in general practice: an observational study in 239 general practices in the Netherlands. BMC Health Serv Res. 2009;9:118.

28. Daugherty SR, Baldwin DC Jr, Rowley BD. Learning, satisfaction, and mistreatment during medical internship: a national survey of working conditions. JAMA. 1998;279(15):1194-9.

29. Oliveira MM. Burnout e emoções: estudo exploratório em médicos de um hospital do Porto [Dissertation]. Porto: Faculdade de Psicologia e Ciências da Educação da Universidade do Porto; 2008. Available from: https://repositorio-aberto.up.pt/handle/10216/25366

30. Nogueira-Martins LA, Jorge MR. Natureza e magnitude do estresse na residência médica [Stress nature and magnitude during medical residency training]. Rev Assoc Med Brasil. 1998;44(1):28-34. Portuguese

31. Melo BT, Gomes AR, Cruz JF. Desenvolvimento e adaptação de um instrumento de avaliação psicológica do burnout para profissionais de psicologia. In: Soares AP, Araújo S, Caires S, editors. Avaliação Psicológica: formas e contextos (Vol. VI). Braga: Associação dos Psicólogos Portugueses; 1999. p. 596-603.

32. Gomes AR, Cabanelas S, Macedo V, Pinto C, Pinheiro L. Stresse, saúde física, satisfação e "burnout" em profissionais de saúde: análise das diferenças em função do sexo, estado civil e agregado familiar. In: Pereira MG, Simães C, Mclntyre T, editors. Modelos, investigação e prática em diferentes contextos de saúde: actas do $2^{\circ}$ Congresso Família, Saúde e Doença, Braga, Portugal, 2008 (Vol. IV). Braga: Universidade do Minho; 2008. p. 178-92.

33. Maslach C, Jackson SE. The measurement of experienced burnout. J Occup Behav. 1981;2(2):99-113.

34. Maroco J, Tecedeiro MM. Inventário de burnout de Maslach para estudantes portugueses. Psic Saúde Doenças. 2009;10(2):227-35.

35. Sá LO. Burnout e controlo sobre o trabalho em enfermagem oncológica: estudo correlacional [Dissertation]. Porto: Faculdade de Medicina da Universidade do Porto; 2002. Available from: https://repositorioaberto.up.pt/handle/10216/9659

36. Marcelino G, Cerveira JM, Carvalho I, Costa JA, Lopes M, Calado NE, et al. Burnout levels among Portuguese family doctors: a nationwide survey. BMJ Open. 2012;2(3):e001050.

37. Legassie J, Zibrowski EM, Goldszmidt MA. Measuring resident wellbeing: impostorism and burnout syndrome in residency. J Gen Intern Med. 2008;23(7):1090-4.

38. Thomas NK. Resident burnout. JAMA. 2004;292(23):2880-9.

39. Schaufeli WB, Bakker AB, Hoogduin K, Schaap C, Kladler A. On the clinical validity of the Maslach burnout inventory and the burnout measure. Psychol Health. 2001;16(5):565-82.

40. Cohen J, Cohen P. Applied multiple regression/correlation for the behavioral sciences. Hillsdale, NJ: Erlbaum; 1983.

41. von Elm E,Altman DG, Egger M, Pocock SJ, Gøtzsche PC, Vandenbroucke JP. The Strengthening the Reporting of Observational Studies in

Epidemiology (STROBE) statement: guidelines for reporting observational studies. J Clin Epidemiol. 2008;61(4):344-9.

42. Brøndt A, Sokolowski I, Olesen F, Vedsted P. Continuing medical education and burnout among Danish GPs. Br J Gen Pract. 2008;58(546): 15-9.

43. Kristensen TS, Borritz M, Villadsen E, Kristensen KB. The Copenhagen Burnout Inventory: a new tool for the assessment of burnout. Work Stress. 2005;19(3):192-207.
44. Embriaco N, Azoulay E, Barrau K, Kentish N, Pochard F, Loundou A, et al. High level of burnout in intensivists: prevalence and associated factors. Am J Respir Crit Care Med. 2007;175(7):686-92.

45. Esteva $M$, Larraz $C$, Jiménez $F$. La salud mental en los médicos de familia: efectos de la satisfacción y el estrés en el trabajo [Mental health in family doctors: effects of satisfaction and stress at work]. Rev Clin Esp. 2006:206(2):77-83. Spanish

46. Mangen MH. Étude du burnout chez les medecins generalistes luxembourgeois [Dissertation]. Paris: Faculté de Médecine, Université Pierre et Marie Curie; 2007.Available from: http://www.cmge-upmc.org/IMG/ pdf/mangen_these_burn_out.pdf

47. Neves AC. Síndrome de burnout nos médicos de família do ACES de Gondomar [Dissertation]. Porto: Instituto de Ciências Biomédicas Abel Salazar, Universidade do Porto; 2011. Available from: https://repositorio-aberto.up.pt/bitstream/10216/83679/2/130621.pdf

48. Schaufeli WB, Martínez IM, Pinto AM, Salanova M, Bakker AB. Burnout and engagement in university students: a cross-national study. J Cross Cult Psychol Stud. 2002;33(5):464-81.

49. Azevedo A, Domingues B, Moura J, Santos L. Estão os internos satisfeitos com o internato de medicina geral e familiar? [Are family medicine trainees satisfied with their training program?]. Rev Port Med Geral Fam. 2014;30(1):24-30. Portuguese

50. Faragher EB, Cass M, Cooper CL. The relationship between job satisfaction and health: a meta-analysis. Occup Environ Med. 2005;62(2): 105-12.

51. Sobecks NW, Justice AC, Hinze S, Chirayath HT, Lasek RJ, Chren MM, et al. When doctors marry doctors: a survey exploring the professional and family lives of young physicians. Ann Intern Med. 1999;130(4 Pt 1):312-9.

52. Sequeira-Carlos J. Internato de MGF: vagas de crise ( $2^{\circ}$ episódio). J Med Fam. 2005;80:36-7.

53. Gaspar D. Medicina geral e familiar, uma escolha gratificante [The family medicine: a gratefull choice]. Acta Med Port. 2006;19(2):133-9. Portuguese

54. Portaria n 45/2015, de 20 de fevereiro. Diário da República. $2^{a}$ série(36).

55. Laranjeira CA. O contexto organizacional e a experiência de estress: uma perspectiva integrativa [An integrated perspective of the organisational context and experience of stress]. Rev Salud Pública. 2009;11(1): 123-33. Portuguese

56. Tarnow-Mordi WO, Hau C, Warden A, Shearer AJ. Hospital mortality in relation to staff workload: a 4-year study in an adult intensive-care unit. Lancet. 2000;356(9225):185-9.

57. Frasquilho MA. Um modelo de intervenção integrada em situações de burnout. Rev Factores Risco. 2015;(37):58-67.

58. Ravindranath D. Stress in the medical profession: an evaluation of premedical students, medical students, and doctors [Internet]. University of Berkeley; 2000 [cited 2009 May 5]. Available from: http://nature.berkeley.edu/classes/es196/projects/2000final/ravindranath.pdf

\section{CONFLITOS DE INTERESSE}

Os autores declaram não ter conflitos de interesse a declarar.

O autor John Yaphe não participou no percurso editorial do manuscrito, apesar de integrar o Conselho Editorial da RPMGF. 
FINANCIAMENTO

O trabalho não foi alvo de qualquer tipo de financiamento externo (incluindo bolsas de investigação).

\section{COMISSÃO DE ÉTICA}

Estudo realizado após parecer favorável da Comissão de Ética da ARS Norte.

\section{ENDEREÇO PARA CORRESPONDÊNCIA}

Paula Mendes

E-mail: paulafm3@gmail.com

Recebido em 14-02-2016

Aceite para publicação em 04-12-2016

\section{ABSTRACT}

\section{STRESS AND BURNOUT IN FAMILY MEDICINE TRAINEES IN THE NORTH OF PORTUGAL: A CROSS-SECTIONAL} STUDY

Objectives: To determine the prevalence of burnout among family medicine trainees in the north region of Portugal and to test possible associations of burnout with sociodemographic and occupational factors and stress.

Type of study: Cross-sectional.

Location: Northern Portugal.

Population: Family medicine trainees.

Methods: We used a convenience sample of the trainees attending meetings of the residency program from 3 November to 11 December 2009. We used the Maslach Burnout Inventory and the Stress in Health Professionals questionnaire and collected sociodemographic and professional data. The prevalence of stress and burnout were calculated and associations between job stress and burnout were tested.

Results: We studied a sample of 210 trainees ( $68 \%$ of the population) of whom $72 \%$ were female. The response rate was $92.5 \%$. Most trainees reported moderate stress $(n=127,60.5 \%)$, especially in training activities and in dealing with patients. The prevalence of burnout in trainees ranged between $8.6 \%(95 \% \mathrm{Cl} 4.8-12.4)$ and $34.3 \%(95 \% \mathrm{Cl} 27.9-40.7)$, depending on the definition used. We found that $29.8 \%(95 \% \mathrm{Cl} 23.6-36.0)$ of trainees had high emotional exhaustion, $17.7 \%(95 \% \mathrm{Cl} 12.5-22.9)$ had high depersonalization and $43.4 \%(95 \% \mathrm{Cl} 36.7-50.1)$ had low personal accomplishment. Depersonalization was significantly higher in men $(p<0.001)$ and among those who did not choose general practice as first choice $(p<0.001)$. Stress scores were significantly associated with all components of the Maslach Inventory (EE $p<0.001$, DP $p<0.001$, and DP $p=0.02$ ). In addition, all six subscales of the stress questionnaire were significantly associated with $\operatorname{EE}(p<0.001)$, five with $\mathrm{DP}(p<0.001)$ and salary with RP $(p<0.01)$.

Conclusions: This study found an estimated prevalence of burnout similar to that found in other studies. This stresses the importance of prevention and intervention strategies for this vulnerable group.

Keywords: Professional burnout; Psychological stress; General practice; Internship. 